\title{
Passive Method for Monitoring Atmospheric Phenomena by Evaluating the Cellular Network Signals
}

\author{
Michal KUBA $^{\mathrm{a}}$, Peter FABO ${ }^{\mathrm{b}, 1}$, Dušan PODHORSKÝc ${ }^{\mathrm{c}}$, Dagmar FAKTOROVÁ ${ }^{\mathrm{a}}$ and \\ Adriana SAVIN ${ }^{\mathrm{d}}$

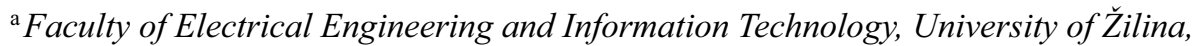 \\ Žilina, Slovak Republic \\ ${ }^{\mathrm{b}}$ Research Centre, University of Žilina, Žilina, Slovak Republic \\ ' Slovak Academy of Sciences, Department of Atmospheric Physics, Bratislava, Slovak \\ Republic \\ ${ }^{\mathrm{d}}$ Nondestructive Testing Department, National Institute of R\&D for Technical Physics, \\ Iasi, Romania
}

\begin{abstract}
An integral part of our environment in addition to the surface of the Earth is the adjacent part of the atmosphere, the lower layer of the troposphere. This part of the troposphere has essential influence on weather evolution and information on its condition is an important part of the weather forecasting models. Standard methods of the data acquisition such as radars, satellites and weather stations do not provide information with sufficiently high temporal and spatial resolution. The subject of the article is a method of monitoring the condition of lower layers of the troposphere by passive monitoring and evaluation the parameters of cellular network signals. The elementary theory of an virtual sensor for the atmospheric radio refractive index monitoring and the preliminary results of experimental monitoring of the selected meteorological situation are presented.
\end{abstract}

Keywords. Index of refraction, radio refractive index, refractivity, radar, satellite, Euler-Lagrange equation

\section{Introduction}

An integral part of our environment is the biosphere which in addition comprise not only of the Earth's surface, but also of the adjacent part of it, the low layers of the troposphere. It is known that about $85 \%$ of the solar energy at the boundary of the atmosphere will hit the Earth's surface at ideal conditions, transforming it to other forms of energy and ultimately to heat. Therefore the condition in the ground layer of the atmosphere has a major influence on the global parameters determining the weather evolution.

The basic parameters for describing the condition of the atmosphere are pressure, temperature, air humidity and the presence of hydrometeors. Because all of these factors affect the propagation of electromagnetic waves, radar measurements from ground stations and satellites have become a standard part of atmospheric monitoring. Because of fundamental reasons, however, they do not affect the ground layers of the atmosphere and do not have sufficient temporal and spatial resolution especially in populated areas where the need for detailed information is highest.

\footnotetext{
${ }^{1}$ Corresponding Author, Peter Fabo, Research Centre, University of Žilina, Žilina 010 26, Slovak Republic; E-mail: peter.fabo@rc.uniza.sk.
} 
The subject of the paper is a description and realization of a passive virtual sensor using specific properties of electromagnetic energy propagation in the atmosphere to describe its refractive index in the radio frequency domain. The determination of refractivity value from temporal parameters of the signal propagating in the atmosphere is described, e.g. in the literature [1], [2]. The basic principle is that the propagation velocity of radio signals depends on the refractive index of the air, which is a function of pressure, temperature, humidity, water vapour density and presence of hydrometeors. Changes in the water vapour density and changes in presence of hydrometeors along the propagation path lead to changes in the propagation velocity. These changes in turn manifests itself in the propagagation delay changes which can finnaly be observed by measuring the changes in the relative phase of the signal between the transmitter and receiver. In contrast to the methods mentioned in the literature [1], [2], the method presented here does not require knowledge of the signal structure, nor autocorrelation parameters of the signals. Also it does not require exact knowledge the value of the time instant at which the signal left the signal source. The determination of the refractive index is based on the measurement of the time parameters in the $1 \mathrm{GHz}$ radio frequency band and advantageously utilizes mobile operators' Base Transceiver Stations (BTS) as energy sources.

In [2] the procedure for continuous tracking the phase of DAB (Digital Audio Bradcasting) signal by pilot symbol tracking is indicated. This algorithm requires some demodulation of the signals to recover the in-built synchronisation (pilot) symbols. The authors had to used a low phase-noise signal generator phase-locked to a GPS disciplined rubidium oscillator to provide a low-jitter ADC sample clock. The DAB signal contains $\mathrm{a} \approx 1 \mathrm{~ms}$ synchronisation symbol in every $\approx 96 \mathrm{~ms}$ data frame. From this a carrier burst can be extracted and the changes in phase can be tracked using a phase-locked-loop or some other form of phase estimator. The carrier burst is recovered from the synchronization symbol by mixing the synchronization symbol with a conjugated replica of the constant-amplitude zero auto-correlation (CAZAC) sequence. The null symbol, which precedes synchronization symbol, consists of repeated CAZAC sequence.

In [1] a roundtrip propagation delay between the receiver and the reflector in form of the phase delay using the phase in the side peak of the delay profile is determined. Delay profiles are defined as the impulse responses of system whose inputs are signals from transmitters and whose outputs are signals received by a receiver. The authors derived the delay profiles by inverse Fourier transform of OFDM (Orthogonal Frequency Division Multiplexing) synchronization symbol in received signals from ISBD-T - the digital terrestrial TV broadcasting standard used in Japan so the algorithm requires some demodulation of the signals as well. By using this method it is possible to measure the variation of propagation delay between the receiver and the reflector in millimeter order.

Unlike the methods described in [1] and [2] the proposed method determines the propagation delay changes in form of the phase delay changes as the time difference in propagation delay between two not very different propagation paths in Line-of-Sight (LOS) toward the BTS in the area between the transmitter/BTS and the receiver without the need of using the reflector. The real-time phase measurement system adopts a software defined radio (SDR) technique. The main components are LimeSDR, which is two channel SDR device supplied by Lime Microsystems, a PC and two commercial Yagi type antennas. The two-channel coherent SDR is essentially two SDRs on a common local oscillator so there is the precise phase synchronisation between the two channels/receivers. Two similar but steel different propagation paths are selected using two directional antennas. These antennas are located in the same place or there may be a 
small distance between them. More about this is discussed in the section 3. In this study only the synchronisation signal of mobile operators in GSM standard which is transmitted by each BTS was utilized because of high penetration of mobile operator's BTSs in the territory of the Slovak Republic. The signals received by the antennas are sampled to produce in-phase and quadrature phase (IQ) time series in each of the two channels. From these two complex time series the phase delay between the two propagation paths is determined without the need for signal demodulation. The advantages of the proposed method are that there is no need to use neither antenna reflector nor high precision external local oscillator and signal demodulation is not required as well. So it is no necessary to use a priori information about time structure of the signal. The disadvantage is the use of two-channel SDR which is more expensive then the single-channel SDR. By using SDR with a high sampling rate (in this case $25 \mathrm{Msps}$ ), it was possible to monitor the entire radio spectrum (from $935 \mathrm{MHz}$ to $960 \mathrm{MHz}$ ) in which the downlink (BTS to mobile) of GSM-900 standard occurs. Because the frequencies of the pilot signals among neighboring BTSs are different from each other, it was possible to measure changes in propagation delay simultaneously from different directions from all BTSs which are surrounding the receiver. In the longer term it is possible using a network of more such receivers deployed over a larger area as a network of passive sensors receiving cellular network signals to measure path-averaged refractivity from different directions and after some processing that information (by using e.g. the tomographic techniques) there might be the possibility to derive two-dimensional, time-evolving boundary-layer moisture fields and spatially strongly bounded meteorological situations related to humidity, rainfall, turbulence etc. in the given area.

\section{Atmospheric refractivity}

The standard methods for monitoring the atmospheric condition focus on monitoring the movement and properties of air masses with the primary goal of obtaining the information which is needed to produce weather forecasts.

The standard methods include the radar measurements, multispectral monitoring of the atmosphere and the Earth's surface by satellites, ground station networks and additional sources of information such as balloon measurements, aircraft and ship crew reports. However, these methods are not suitable for determination the atmospheric condition in the ground layer for height up to the level of $100 \mathrm{~m}$ which significantly affects the environment. The lowest height level of observability by standard meteorological radar usually does not drop below $600 \mathrm{~m}$ in flat areas, satellites usually are not able to observe relatively small local atmospheric changes and the ground station network is relatively sparse and usually covers only critical infrastructure such as airports and ports.

Atmospheric monitoring usually uses the SHF (3-30 GHz) frequency bands and higher, where the attenuation properties of the atmosphere are already present and the wavelength allows monitoring of hydrometeors and their manifestations (Doppler effects, polarization and others). In the frequency band below $3 \mathrm{GHz}$ the attenuation effects of hydrometeors is practically negligible [3] therefore these frequency bands are not suitable for use by standard methods. However the parameter that affects the propagation of electromagnetic energy in the atmosphere over the whole frequency range is the refractive index of the atmosphere which determines the propagation velocity of 
electromagnetic waves. The typical value of refractive index for a standard atmosphere is typically of $n=1.000320$ which slightly differs from the value of 1 . However, for practical reasons the index of refractivity is defined as follows:

$$
N=(n-1) * 10^{6}
$$

For average value of refractivity as a function of height $h[\mathrm{~km}]$ (long-term average value for the globe, $h_{0}=7.35 \mathrm{~km}$ ) according to [4], [5] the following relationship holds:

$$
N=N_{0} \cdot \exp \left(-\frac{h}{h_{0}}\right)=315 \cdot \exp \left(-\frac{h}{7.35}\right)
$$

According to [6] for low altitudes the approximate value of $-39 \mathrm{~N} / \mathrm{km}$ of vertical gradient of refractivity usually is used.

\section{Principle of refractivity measurement}

The principle of a virtual sensor operation is shown on elementary theory in the simplest case of a linear vertical dependence of refractive index, which can be defined in this form:

$$
n(y)=n_{0}(1+\alpha y)
$$

where $n_{0}$ is the refractive index on the Earth's surface, $\alpha$ is the gradient value. For such a refractive index profile we look for a trajectory $y(x)$ along which electromagnetic energy is propagated in the environment between the source and the detector. The optical length of the trajectory (terminology from the optics) is given as:

$$
f\left(x, y, y^{\prime}\right)=\int n \mathrm{dl}=\int n(y) \sqrt{1+\left(y^{\prime}\right)^{2}} \mathrm{dx}
$$

The trajectory $y(x)$ is obtained by solving the Euler-Lagrange differential equation:

$$
\frac{\mathrm{d}}{\mathrm{dx}}\left(\frac{\partial f}{\partial y^{\prime}}\right)-\frac{\partial f}{\partial y}=0
$$

in form of:

$$
y(x)=-\frac{1}{\alpha}+\left(y_{0}+\frac{1}{\alpha}\right) \cosh \left(\frac{x}{y_{0}+\frac{1}{\alpha}}\right)
$$

where $y_{0}$ is the value determined from the initial conditions as the highest point of the trajectory. For example, in Fig. 1 the calculated trajectories for a vertical gradient of $-40 \mathrm{~N} / \mathrm{km}$ are depicted. The trajectory endpoints are 900 and $1000 \mathrm{~m}$ away from the signal source. 
The goal is to determine the time difference between the propagation times of the signal from the source to the destination points A and B for the different refractivity gradient values. The optical length of the SA trajectory is given as

$$
L_{S A}=\int_{x_{S}}^{x_{A}} n(y) \mathrm{ds}=\int_{x_{S}}^{x_{A}} n_{0}(1+\alpha y(x)) \sqrt{1+y^{\prime}(x)} \mathrm{dx}
$$

Similarly the optical length of the SB trajectory can be obtained. Let $\lambda$ is the wavelength of the source of the electromagnetic energy. Because it seems that the time variations will be very small it is reasonable rather to work with phase delay $\phi$ instead of using the time difference between signals at points A and B.

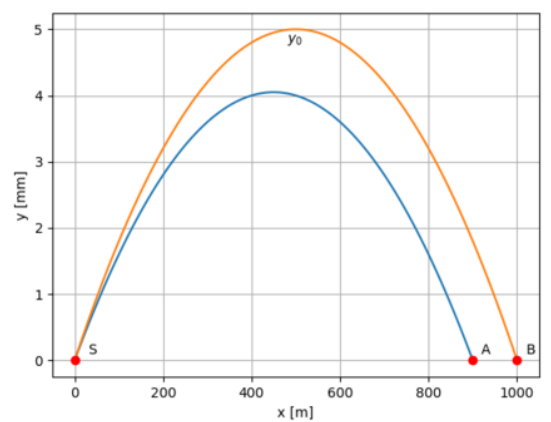

Figure 1. The trajectory between the source and the two destination points A and B.

The $L_{S A}$ and $L_{S B}$ optical lengths are functions of refractivity and its gradient. The $L_{A B}$ distance between the points $\mathrm{A}$ and $\mathrm{B}$ does not depend on the refractivity and gives a constant offset to the result. Therefore it can be subtracted from the difference of optical lengths:

$$
\phi=\frac{L_{S B}-L_{S A}-L_{A B}}{\lambda} \cdot 2 \pi
$$

After all numerical calculations of integrals which define the values of $L_{S A}$ and $L_{S B}$ optical lengths and the signal phase delay value, the distance between antennas in meters was selected when the refractive index value varies approximately in the range from 290 to 400 .

\section{Experimental results}

It is clear that by measurement of the phase delay between signals at points A and B it is possible to obtain values which are proportional to the atmospheric refractivity. There is obvious relationship among physical parameters of the atmosphere and its refractivity. Therefore, it is possible to obtain a virtual sensor for monitoring the condition of the atmosphere on the SAB line in a such relatively simple manner.

A direct comparison of the phase delay and refractivity determined from standard meteorological sensors (pressure, temperature, humidity) is, for obvious reasons, 
however, only possible for specific meteorological situations in which the atmosphere is homogeneous along the path of propagation which includes the position of the sensors. On Fig. 2 the measured signal phase delay together with the refractivity values, which were calculated from the values obtained from the local measurement of temperature, pressure and humidity is depicted.

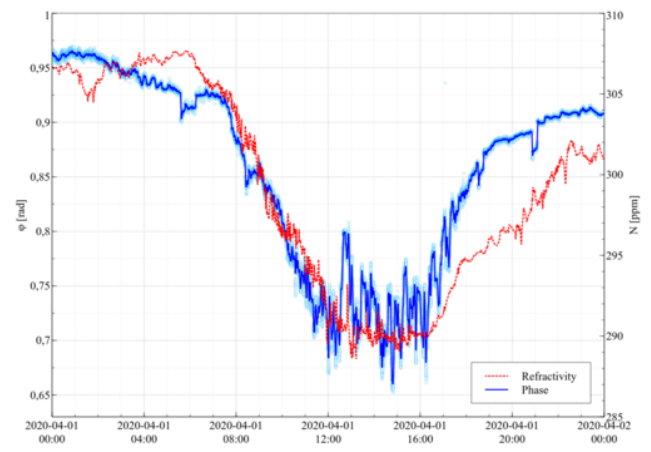

Figure 2. The comparison of the phase delay and the refractivity.

Fig. 3 shows the results obtained by monitoring of cold front passage over a region of western Slovakia along with radar measurements at indicated times and areas. The measurements were taken in Trenčín city, the distance to the BTS was $400 \mathrm{~m}$, the distance between the antennas at points A and B was approximately $2 \mathrm{~m}$. Several BTS were monitored simultaneously in receiver bandwidth of $25 \mathrm{MHz}$.

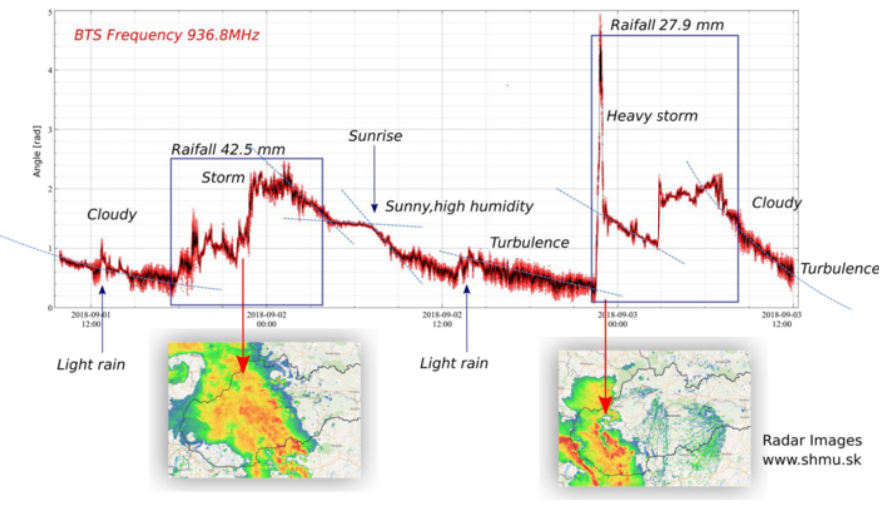

Figure 3. Cold front passage monitoring

The monitored BTS is operated at a frequency of $936.8 \mathrm{MHz}$. The GSM G2 mobile network pilot signal was being received. The total bit rate from the dual channel SDR was $320 \mathrm{MB} / \mathrm{s}$, the total amount of processed data in the figure was approximately $20 \mathrm{~TB}$ at a recording time of approximately 2 days.

The data show the effect of atmospheric phenomena on the phase angle - rainfall and storms, the relaxation of atmospheric humidity after rainfall, the influence of the sun, humidity changes during the day, the origin and extinction of thermal turbulence as well as the extinction of vertical atmospheric movements at night. 


\section{Conclusion}

From the practical point of view the measurement requires using an arbitrary two-channel coherent SDR receiver with sufficiently fast enough communication interface and a computer for evaluation of the phase shift between IQ signals from both channels of the receiver. As a signal sources the BTS of mobile operators in the LOS were used. At the same time a more signals can be received if the sufficiently powerful SDR receiver will be used and therefore it is possible to monitor the condition of the atmosphere from different directions. From mentioned above the following features of the technology result:

- $\quad$ passive method - no self-radiation - utilizes radiation from another sources

- without legislative restrictions - no data streams decoding

- creates virtual sensors among power sources and receiving antennas

- possibility of measurements from more directions simultaneously

- $\quad$ independence on modulation formats, type of service, etc.

Description and implementation of the above mentioned virtual sensor is one of the many possibilities of monitoring the condition of the lower layers of the troposphere by passive monitoring and evaluation of the sources of electromagnetic radiation on the Earth's surface. Another method that have been successfully tested in the project utilizes the correlation properties of the power characteristics of spatial and diversity reception. The monitoring of signal sources can be extended to multiple receiving systems. This way can be obtained the system of many intersecting LOS and by using of the suitable algorithms there is the possibility to obtain the information about spatial localization of the selected meteorological phenomena such as local rainstorms and downpours, ground fog areas and others.

\section{Acknowledgments}

This work was supported by grant of the Scientific Grant Agency of the Slovak Republic, VEGA 2/0015/18 Meso- and micro-meteorological exploration of the occurrence of hydrometeors in boundary layer of troposphere based on passive evaluation of changes of electromagnetic radiation from anthropogenic sources.

This work was supported by grant of the Slovak Research and Development Agency APVV-17-0218 and Romanian Ministry of Research and Innovation - CCCDI UEFISCDI Project 11PFE/2018.

\section{References}

[1] S. Kawamura et al., Water vapor estimation using digital terrestrial broadcasting waves, Radio Science, Volume 52, Issue 3, March 2017, Pages: 283-427.

[2] R.J. Watson and Chris Coleman, The use of signals of opportunity for the measurement of atmospheric refractivity, Conference: Antennas and Propagation (EuCAP), 2010, Proceedings of the Fourth European Conference on, Pages: 1-5.

[3] C.C. Chen, Attenuation of Electromagnetic Radiation by Haze, Fog, Clouds and Rain, United States Air Force Project RAND, 1975. 
[4] B.R. Bean, E. Dutton, Radio Meteorology, Central Radio Propagation Laboratory, National Bureau of Standards, Boulder, Colorado, 1966.

[5] ITU-R, The radio refractive index: its formula and refractivity data, ITU 2016.

[6] A.R. Webster, Raypath Parameters in Tropospheric Multipath Propagation, IEEE Transactions on Antennas and Propagation, vol. AP-30, no. 4, Jul. 1982, pp. 796-800. 